\title{
Stepped Propane Adsorption in Pure-Silica ITW
}

\section{Zeolite}

Jung Gi Min, ${ }^{\dagger}, \|$ Azahara Luna-Triguero, ${ }^{\ddagger}, \|$ Youngchul Byun, ${ }^{\dagger}$ Salvador R. G. Balestra,${ }^{\ddagger}$ Jose Manuel Vicent-Luna, ${ }^{\ddagger}$ Sofia Calero, ${ }^{*}{ }^{\ddagger}$ Suk Bong Hong,,${ }^{\dagger \dagger}$ and Miguel A. Camblor ${ }^{*}$,

${ }^{\dagger}$ Center for Ordered Nanoporous Materials Synthesis, Division of Environmental Science and Engineering, POSTECH, Pohang 37673, Korea

Department of Physical, Chemical and Natural Systems, Universidad Pablo de Olavide, Crtra. De Utrera km. 1, 41013 Seville, Spain.

${ }^{\S}$ Instituto de Ciencia de Materiales de Madrid (ICMM), Consejo Superior de Investigaciones Científicas (CSIC), Sor Juana Inés de la Cruz 3, 28049 Madrid, Spain

"These authors contributed equally.

\section{Corresponding Author}

*scalero@upo.es; *sbhong@postech.ac.kr; *macamblor@icmm.scic.es 
ABSTRACT: Gas adsorption over zeolites is at the basis of important applications of this class of microporous crystalline solids, notably as separation media and catalysts, but it may also be complex and not straightforward to understand. Here we report that for temperature below $323 \mathrm{~K}$ propane adsorption on the small-pore pure-silica zeolite ITW exhibits a clear step (pseudosaturation). This is absent in the case of propene and the other small linear alkanes. An intermediate plateau, clearly observed in the $293 \mathrm{~K}$ isotherm, always occurs when one molecule of propane is loaded in every other cage, i.e., at half saturation. The simulation results show a swelling of the ITW structure upon propane adsorption. The strong dependence of available pore volume on the adsorbate loading level implies that adsorption cannot occur on the void structure while saturation can only be reached on highly loaded structures. To account for this unprecedented adsorption phenomenon, we propose the term "guest-modulated effect".

KEYWORDS: adsorption $\cdot$ inflection $\cdot$ propane $\cdot$ computational chemistry $\cdot$ zeolite ITW 


\section{INTRODUCTION}

The large surface areas and well-defined pore structures of zeolites, together with their high surface selectivities to certain gases that depends mainly on the framework composition, determine their possible applications. ${ }^{1,2}$ It has long been recognized that the adsorption isotherms on these crystalline materials can present inflections or steps, implying that pseudo-saturation levels are reached before further increase in the relative pressure triggers more adsorption and finally saturation. Müller et $a l^{3}$ were the first to observe a step in the adsorption of $\mathrm{N}_{2}$ and $\mathrm{Ar}$ on the medium-pore high-silica $(\mathrm{Si} / \mathrm{Al}>1000)$ zeolite ZSM-5. This behaviour has been attributed to the initial filling of channels followed by the filling of channel intersections, with indication of a transition of the adsorbate from a fluid-like to a solid-like phase. By contrast, the step found during $\mathrm{Kr}$ adsorption on pure-silica ZSM-5 was considered to be due to 'the completion of the monolayer', ${ }^{4}$ while the stepped isotherms of other small gases like Ar and $\mathrm{CO}$ have been related to a disorder-order, fluid-to-crystalline transition in the adsorbed phase. ${ }^{5,6}$ This phase transition was suggested to be analogous to the spinodal decomposition involving a metastable liquid-like phase where the adsorbed molecules have a restrained mobility in the confined pore space and relax only under excess pressure to a more stable solid-like adsorbed phase. ${ }^{7}$

On the other hand, the step in the adsorption of toluene on ZSM-11, another medium-pore zeolite, has been attributed to the selective adsorption at different sites (first the intersections followed by the channels), without a clear evidence for an adsorbate transition. ${ }^{8}$ A more interesting case occurs when linear alkanes of intermediate length ( $n$-hexane and $n$-heptane), are adsorbed on ZSM-5. ${ }^{9}$ This is not found for shorter or longer alkanes. The observed steps have been explained by a commensurate freezing effect of the molecules in the sinusoidal ten-membered ring channels (10MR, windows built from ten $\mathrm{TO}_{4}$ tetrahedra, where $\mathrm{T}$ is $\mathrm{Si}$ or $\mathrm{Al}$ ). This allows further loading 
and that is possible only when the length of adsorbate molecules is similar to the sinusoidal channel period. ${ }^{10}$ Stepped adsorption can also be observed for the small linear alkanes on metal-organic frameworks (MOFs). For instance, the steps appearing in the adsorption isotherms of methane, ethane, propane, and $n$-butane in MIL-53(Fe) have been ascribed to the existence of four discrete pore opening states as a function of alkane pressure. ${ }^{11}$ The known flexibility of this class of hybrid porous materials was invoked to account for the steps by the breathing effect associated with structural transitions between different states of pore openings. ${ }^{12-14}$

The small-pore zeolite ITW possesses obloid slit-shaped cavities that are connected through 8MR pore openings, yielding pores wide enough to allow the passage of small alkane molecules only along the crystallographic $z$-axis. ${ }^{15}$ There is a second connection through $8 \mathrm{MR}$ windows between cavities along the $x$-axis that is, however, too narrow even for methane, the smallest

hydrocarbon, with a kinetic diameter of $3.8 \AA{ }^{16}$ Thus, the void space of ITW should be described as a one-dimensional (1D) cage-based pore system with regard to the adsorption of small linear alkanes. Pure-silica ITW has been reported to selectively adsorb propene over propane, rendering it very attractive as an adsorbent for the non-cryogenic separation of the same species from the mixtures with propane. ${ }^{17}$ Here we report the presence of a clear step in the adsorption isotherms of propane in pure-silica ITW. This step is not observed for propene. Our simulation results allow us to understand the stepped propane adsorption in this small-pore zeolite.

\section{EXPERIMENTAL DETAILS}

Adsorption Isotherm Experiments. The propane (99.99\%, Linde) adsorption isotherms for the pure-silica calcined ITW sample with an Ar BET surface area of $311 \mathrm{~m}^{2} \mathrm{~g}^{-1}$ were performed volumetrically in a temperature range $263-323 \mathrm{~K}$ and at a pressure range $0.0-2.0$ bar using a 
Setaram PCTPro E\&E analyzer. Prior to the experiments, $300 \mathrm{mg}$ of sample were placed into the stainless sample holder (height, $21 \mathrm{~mm}$; o.d., $2.0 \mathrm{~mm}$; i.d., $1.7 \mathrm{~mm}$ ) and evacuated under vacuum to a residual pressure of $10^{-3}$ Torr at $523 \mathrm{~K}$ for $6 \mathrm{~h}$. Propane was added incrementally and data points were recorded when no further change in uptake was observed $\left(<2.0 \times 10^{-6} \mathrm{wt} \% \mathrm{~min}^{-1}\right)$. Because of the extremely slow propane adsorption kinetics on ITW, the experimental time to complete the isotherms was exceedingly long, reaching a maximum of approximately 720 hours (1 month). The adsorption isotherms of methane (99.995\%), ethane (99.95\%), propene (99.95\%) and n-butane (99.95\%), all of which were obtained from Linde, were also collected at $293 \mathrm{~K}$ in a manner similar to that described above. When necessary, high-pressure isotherms up to 20 bar were measured.

Computational Methods and Models. The ITW structures with several propane loadings, from empty to saturated, were minimized using Baker's method in the RASPA software. ${ }^{18,19} \mathrm{We}$ performed simulations in NoT ensemble using a fully-flexible unit cell that allowed variations for the box lengths, $a, b, c$, as well as for the angle $\beta$. However, $\alpha=\gamma=90^{\circ}$ are fixed to keep the monoclinic crystal system. The minimizations used the well-known core-shell potential of Sander et al. ${ }^{20}$ where increasing the loading of adsorbates yielded a set of different structures. To study the variation in the structure as a function of loading with different guest molecules, we performed minimizations in the same conditions using propene. Adsorbates are described using the united atom models reported by Dubbeldam et al. for alkanes and by Liu et al. for alkenes. ${ }^{21-23} \mathrm{The}^{\mathrm{CH}} \mathrm{H}_{3}$ $\left(s p^{3}\right), \mathrm{CH}_{2}\left(s p^{3}\right.$ and $\left.s p^{2}\right)$ and $\mathrm{CH}\left(s p^{2}\right)$ groups are considered as non-charged single interaction centres with their own effective potentials. Intra- and intermolecular parameters were taken from the literature. ${ }^{21-23}$ The adsorption isotherms of propane were computed for the minimized ITW structures. During adsorption, the structures were modelled as rigid with the framework atoms at 
crystallographic positions. The adsorption isotherms were calculated using Monte Carlo in the grand canonical ensemble (GCMC) where chemical potential, volume and temperature are fixed. Pressure was obtained from fugacity using the Peng-Robinson equation. ${ }^{24}$ We used $20^{5} \mathrm{MC}$ cycles after $10^{4}$ cycles of initialization. MD simulations in the NVT ensemble were performed to calculate the diffusion of hydrocarbons in ITW. We use a single molecule of hydrocarbon randomly located in the simulation box. Simulations were performed at several temperatures (from 293 to $623 \mathrm{~K}$ ), fixed using the Nose-Hoover thermostat. ${ }^{25,26} \mathrm{We}$ used $10^{9} \mathrm{MD}$ cycles for the production run and time step intervals of 2 fs after $10^{5}$ equilibration cycles.

\section{RESULTS AND DISCUSSION}

Pure-silica ITW was synthesized and activated according to the procedures given elsewhere. ${ }^{15}$ The powder X-ray diffraction and Ar adsorption results reveal that it is highly crystalline and phase-pure (Figure S1). The analysis of the pore system using Zeo++, M. Haranzyk's program based on a Voronoi decomposition, ${ }^{27}$ estimates the limiting apertures along both $\mathrm{z}$ and $\mathrm{x}$ axes as 4.1 and $2.1 \AA$, respectively. The propane adsorption isotherms on our ITW for the temperature range 263 - $323 \mathrm{~K}$ show a step at temperatures lower than $308 \mathrm{~K}$ (Figure 1a). Details on the adsorption isotherm experiments can be found in supporting information. Regardless of the adsorption temperature, the first plateau with a capacity of $0.61 \mathrm{mmol} \mathrm{g}^{-1}$ appears at pressures below 1.0 bar. This pseudo-saturation plateau corresponds to one molecule of propane loaded into half of the cages in the ITW channel system. Further increase in the pressure of propane triggers 

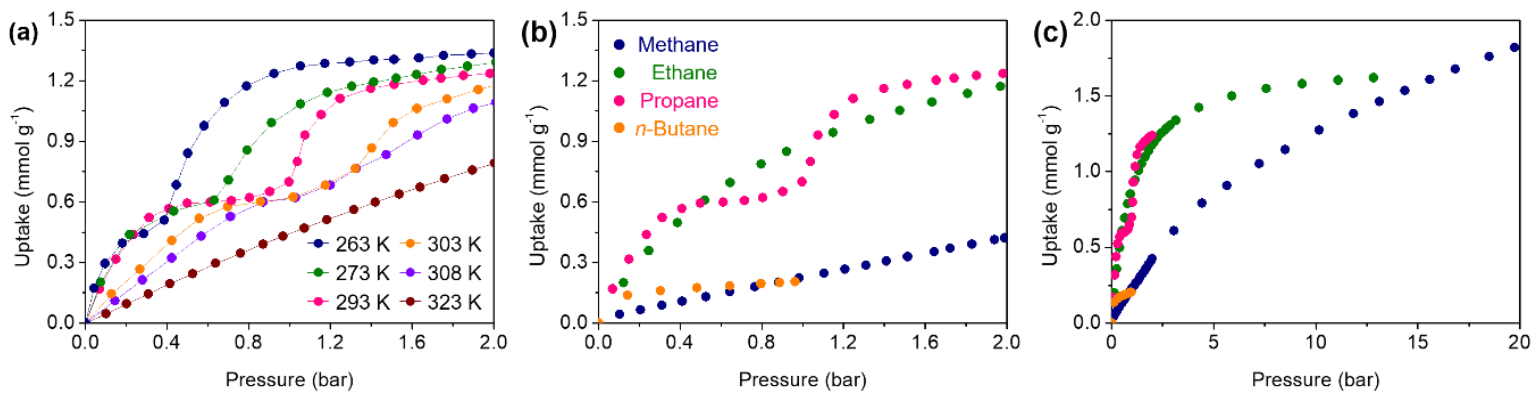

Figure 1. (a) Low-pressure adsorption isotherms of propane at $263-323 \mathrm{~K}$ and $0-2.0$ bar. (b) Low- and (c) high-pressure adsorption isotherms of methane, ethane, propane and n-butane at 293 $\mathrm{K}$ and pressures up to 2.0 and 20 bar on pure silica ITW zeolite. Each data point is recorded when no further change in uptake was observed $\left(<2.0 \times 10^{-6} \mathrm{wt} \% \mathrm{~min}^{-1}\right)$. Due to extremely slow propane adsorption kinetics on this zeolite, the measurement was performed for a maximum of approximately 720 hours (1 month) to obtain one single isotherm.

the steep increase in adsorption, with the final saturation plateau corresponding to a capacity that is almost twice the value of the first plateau. The second saturation thus accounts for a full occupancy of the cavities, i.e., one propane molecule per cavity. We also found that as the temperature increases from 263 to $293 \mathrm{~K}$, the adsorption isotherms essentially superimpose before the pseudo-saturation plateau (Figure 1a). However, above $293 \mathrm{~K}$, the adsorption decreases with temperature. This makes the plateau more markedly visible around $293 \mathrm{~K}$, more diffuse at higher temperatures and ultimately lost at $323 \mathrm{~K}$. Therefore, the additional pressure needed to induce the second adsorption process increases with temperature.

A commensurate freezing effect in the effectively 1D pore system cannot possibly open a second pore system for further adsorption, unlike the case of ZSM-5 with two intersecting 10MR channels. Also, adsorbate-adsorbate interactions are quite limited since each cavity is occupied by 

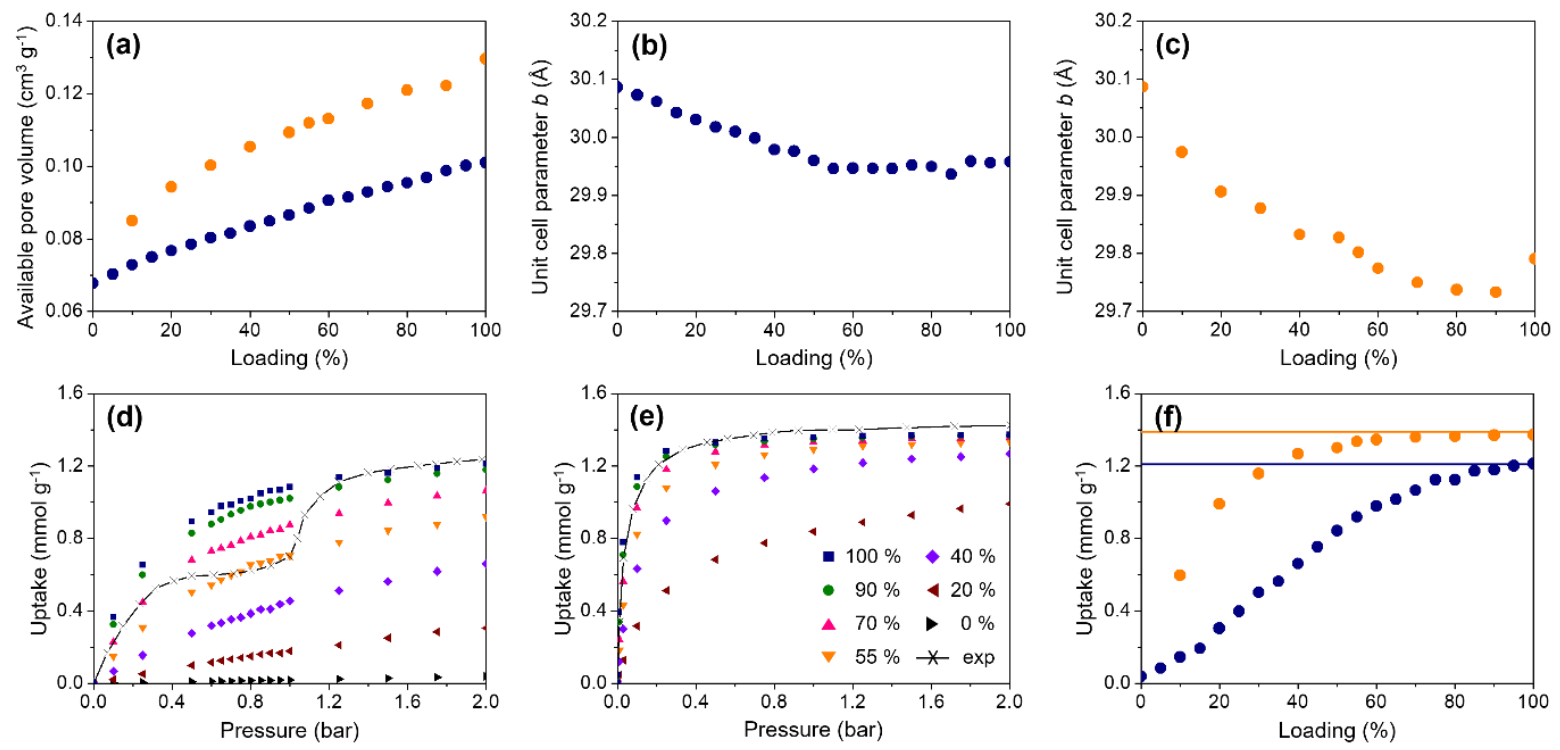

Figure 2. (a) Available pore volume of the ITW structure minimized as a function of propane (navy dots) and propene (orange dots) loading percentages (from zero to 100\%). Variation in the unit cell parameter $\mathrm{b}$ as a function of (b) propane and (c) propene loading percentages. Simulated adsorption isotherms in the minimized ITW structures at $293 \mathrm{~K}$ for (d) propane and (e) propene. Experimental isotherms (barbed lines) are included for comparison. (f) Uptakes for propane (navy) and propene (orange) at $293 \mathrm{~K}$ and $2.0 \mathrm{bar}$ as a function of their loading percentages on the minimized ITW structures. Solid lines indicate the experimentally determined values.

at most one molecule. Therefore, the adsorbate phase transitions in a system of truly isolated adsorbate molecules can in principle be ruled out. The fact that ITW is formed by one single type of cavities and one single type of pores cannot explain the observed steps, either. These arguments led us to conclude that the origin of the stepped propane adsorption in this small-pore zeolite is fundamentally different from any other reported stepped adsorptions in zeolites and MOFs. We also measured the adsorption isotherms of methane, ethane, and $n$-butane in ITW, but the step is not present there, even at the highest values of pressure (Figure 1b,c). The low adsorption capacity observed for $n$-butane is expected, given its kinetic diameter $(4.7 \AA) .{ }^{16}$

To understand the unexpected stepped adsorption of propane in pure-silica ITW, we have carried out molecular simulations using three structures with monoclinic symmetry. These structures were taken from the International Zeolite Association (IZA) website, ${ }^{28}$ experimentally 
determined by Rojas et al. ${ }^{29}$ and geometrically distance-least-squares (DLS) optimized by Gutiérrez-Sevillano et al. ${ }^{30}$ using the RASPA software. ${ }^{18,19}$ Although their structural differences are minor, the adsorption in these three ITW structures varies much. The adsorption is almost null in the experimental structure and full or almost full for the DLS-optimized structure and IZA structures, respectively (Figure S2). This suggests that propane adsorption on ITW is strongly altered by subtle changes in the structure.

The calculated adsorption isotherms for methane and ethane in the DLS-optimized structure at $293 \mathrm{~K}$ and 0-2 bar agree with the experimental isotherms. This validates the models and force fields used here. However, there is substantial disagreement between experimental and simulation results for propane and $n$-butane (Figure S3). The calculated adsorption for $n$-butane is overestimated because the Monte Carlo simulations are not taking into account kinetics. In the case of the adsorption of propane, the calculated loadings did not reflect the experimental step around 1.0 bar either. The freezing effect is not a plausible option since ITW lacks channel intersections, so we explored the possibility of adsorption modulated by a guest-induced framework relaxation. To this aim, we performed ITW structure minimizations as a function of propane and propene loadings, starting with the bare structure and progressively adding adsorbates up to saturation. Consequently, we obtained a large number of minimized structures and calculated their pore size distributions (PSDs) and accessible pore volumes.

Considering the similarities in the kinetic diameter of propane $(4.3 \AA)$ and propene $(4.5 \AA),{ }^{16}$ one may expect that the minimised structures are independent of the type of adsorbate used. However, the available pore volume is always larger for the propene-loaded structures (Figure 2a). For instance, the structure with a propene loading level of $40 \%$ was calculated to have an available pore volume of $0.10 \mathrm{~cm}^{3} \mathrm{~g}^{-1}$. To reach equivalent pore volume, the ITW structure should be 
completely saturated with propane. Therefore, the interactions with the zeolite framework are stronger for propene than for propane, explaining the well-known outstanding propene selectivity of this small-pore zeolite. ${ }^{17}$ On the other hand, the unit cell parameters $a, c$, and $\beta$ of the ITW structure increase or decrease with increasing loading level of both propane and propene, although their variations are larger for the latter olefin (Figure S4). It is also remarkable that the unit cell length $b$ initially decreases from $30.09 \AA$ (bare structure) to $29.94 \AA$ with increasing propane loading up to $55 \%$ and then remains essentially constant at higher loadings, unlike the case of propene loading (Figure 2b,c). Such variations in unit cell parameters lead to global transformation of structures, thus affecting their PSDs: the most intense peak for propane moves from 3.6 to 4.0 $\AA$ for propane and from 3.6 to $4.5 \AA$ for propene (Figure S5). When the structure is filled by $20 \%$ with propene this peak is at $4.2 \AA$.

We next calculated the adsorption isotherms of propane in all minimized ITW structures using Monte Carlo in the grand canonical ensemble ${ }^{31}$ where chemical potential, volume and temperature are fixed. At the pressure range studied here, propane cannot enter the bare structure (Figure 2d). Although the zeolite structural changes upon loading are tiny, the effects on adsorption are huge. The experimental adsorption isotherm of propane consist of two regions. The first region goes up to the step at about 1 bar, and the second region follows up to saturation (at about 2 bar). The first region of the isotherms is well reproduced using the ITW structure minimized with a propane loading level of 55\%. This is also the structure that provides the inflection point in the unit cell parameter $b$. The second region is reproduced using the most expanded ITW structure, minimized with $100 \%$ propane loading. Therefore, the stepped adsorption of propane in this small-pore zeolite can be rationalized by a guest-modulated adsorption in which the available pore space, and hence the apparent saturation, strongly depends on molecular loading. 

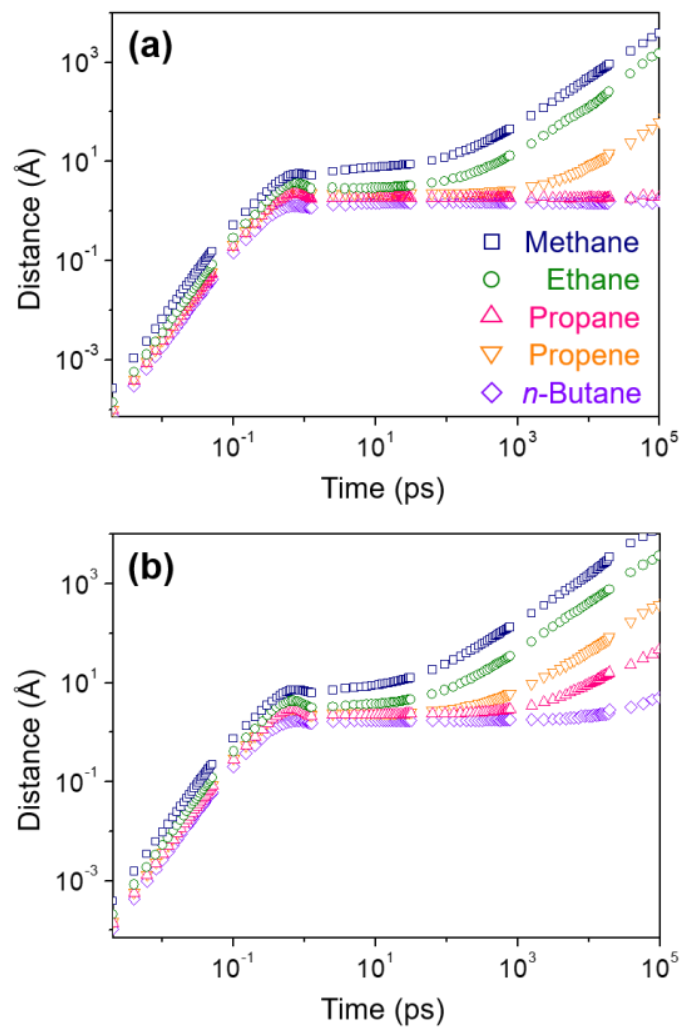

Figure 3. Mean square displacements as a function of time for one single molecule of methane, ethane, propane, propene, and $n$-butane in ITW at (a) 423 and (b) $623 \mathrm{~K}$.

Using the same method described above, we also calculated the adsorption isotherms of propene in ITW structures. Unlike for propane adsorption, all the structures minimized with loading levels larger than 40\%, can reach saturation (Figure 2e). However, only the largest structures (minimized with loading levels above $80 \%$ ) provide the experimental saturation values. (Figure 2f). Both propane and propene exert a loading effect in the ITW structure. This is cooperatively dynamic in nature and enhances adsorption upon loading. The propene sorptionenhancement effect appears to be strong enough to give a smooth isotherm, whereas the plateau in the adsorption isotherm of propane could be considered a pseudo-saturation induced by a less efficient structural swelling that effectively modulates adsorption ("guest-modulated adsorption"). 
The self-diffusion coefficients $\left(D_{\mathrm{S}}\right)$ of small hydrocarbons in ITW was reported to be $10^{-16}$ $10^{-14}$ at $298 \mathrm{~K}^{30}$ Such extremely low $D_{\mathrm{S}}$ values cannot be obtained from the mean square displacements (MSDs), being necessary to perform molecular dynamics simulations, using one single adsorbate (methane, ethane, propane, propene, and $n$-butane) inside the ITW structure at higher temperatures. Figure 3 a shows the $D_{\mathrm{S}}$ values obtained from the MSDs for methane, ethane, and propene at $423 \mathrm{~K}$. The $D_{\mathrm{S}}$ values obtained for methane and ethane at $298 \mathrm{~K}$ are also available in the Figure S6. However, much higher temperature (e.g., $623 \mathrm{~K})$ was required to calculate the $D_{\mathrm{S}}$ value for propane (Figure $\left.3 \mathrm{~b}\right)$. Since low-pressure (<1.0 bar) propane adsorption experiments required extremely long time (ca. 200 hours) to reach equilibrium, it appears that some energy may be needed to expand the ITW framework during adsorption.

\section{CONCLUSIONS}

In summary, we found a clear stepped propane adsorption on the pure-silica zeolite ITW at temperatures below $323 \mathrm{~K}$. Other small linear alkanes, including propene, show simple and smooth isotherms. The first plateau (pseudo-saturation) always turns when one molecule of propane occupies every other cage in ITW. The computer simulation results reveal that the available pore volume of this small-pore zeolite depends strongly on the loading level of propane. We propose the term "guest-modulated effect" to explain this previously unknown adsorption phenomenon.

\section{ASSOCIATED CONTENT}

Supporting Information 
The Supporting Information is available free of charge on the ACS Publications website at DOI: $\mathrm{xx} . \mathrm{xxxx} / \mathrm{xxxxxx}$.

Characterization results, sorption simulation, structural changes in the unit cell parameters, structure optimization, diffusion calculation

\section{AUTHOR INFORMATION}

\section{Corresponding Authors}

*E-mail: scalero@upo.es (S.C.).

*E-mail: sbhong@postech.ac.kr (S.B.H.).

*E-mail: macamblor@icmm.scic.es (M.A.C.).

ORCID

Jung Gi Min: 0000-0002-4084-1192

Azahara Luna-Triguero: 0000-0001-9936-3802

Salvador R.G. Balestra: 0000-0002-2163-2782

Jose Manuel Vicent-Luna: 0000-0001-8712-5591

Sofia Calero: 0000-0001-9535-057X

Suk Bong Hong: 0000-0002-2855-1600

Miguel A. Camblor: 0000-0001-9591-3432

\section{Author Contributions}

"J.G.M. and A.L.: these authors contributed equally.

Notes

The authors declare no competing financial interest. 


\section{ACKNOWLEDGMENTS}

This work was supported by Spanish Ministerio de Economía y Competitividad (CTQ201680206-P, MAT2015-71117-R) and the National Creative Research Initiative Program (2012R1A3A-2048833) through the National Research Foundation of Korea. The authors also thank Dr. R. Ruiz-Salvador for guidance with optimization techniques for flexible zeolites with guest molecules. S.R.G.B. thanks the "Ministerio de Economía y Competitividad" for his predoctoral fellowship. 


\section{REFERENCES}

(1) Davis, M. E. Ordered porous materials for emerging applications. Nature 2002, 417, 813821.

(2) Camblor, M. A.; Hong, S. B. Synthetic silicate zeolites: diverse materials accessible through geoinspiration. In porous materials; Bruce, D. W.; O’Hare, D.; Walton, R. I. Wiley: Chichester, U.K., 2011, pp. 265-325.

(3) Müller, U.; Reichert, H.; Robens, E.; Unger, K. K.; Grillet, Y.; Rouquerol, F.; Rouquerol, J.; Pan, D.; Mersmann, A. High-resolution sorption studies of argon and nitrogen on large crystals of microporous zeolite ZSM-5. Z. Anal. Chem. 1989, 333, 433-436.

(4) Hope, A. T. J.; Leng, C.; Catlow, C. R. A. The sorption of krypton in silicalite. Proc. R. Soc. Lond. A 1989, 424, 57-79.

(5) Llewellyn, P. L.; Coulomb, J. P.; Grillet, Y.; Patarin, J.; Lauter, H.; Reichert, H.; Rouquerol, J. Adsorption by MFI-type zeolites examined by isothermal microcalorimetry and neutron diffraction. 1. Argon, krypton, and methane. Langmuir 1993, 9, 1846-1851.

(6) Llewellyn, P. L.; Coulomb, J. P.; Grillet, Y.; Patarin, J.; Andre, G.; Rouquerol, J.; Adsorption by MFI-type zeolites examined by isothermal microcalorimetry and neutron diffraction. 2. Nitrogen and carbon monoxide. Langmuir 1993, 9, 1852-1856.

(7) Webb, S. W.; Conner, W. Sorption of gases on microporous solids: pore size characterization by gas sorption. Stud. Surf. Sci. Catal. 1991, 62, 31-40.

(8) Sánchez-Gil, V.; Noya, E. G.; Sanz, A.; Khatib, S. J.; Guil, J. M.; Lomba, E.; Marguta, R.; Valencia, S. Experimental and simulation studies of the stepped adsorption of toluene on pure-silica MEL zeolite. J. Phys. Chem. C 2016, 120, 8640-8652.

(9) Richards, R. E.; Rees, L. V. C. Sorption and packing of $n$-alkane molecules in ZSM-5. 
Langmuir 1987, 3, 335-340.

(10) Smit, B.; Maesen, T. L. M. Commensurate 'freezing' of alkanes in the channels of a zeolite. Nature 1995, 374, 42-44.

(11) Llewellyn, P. L.; Horcajada, P.; Maurin, G.; Devic, T.; Rosenbach, N.; Bourrelly, S.; Serre, C.; Vincent, D.; Loera-Serna, S.; Filinchuk, Y.; Férey, G. Complex adsorption of short linear alkanes in the flexible metal-organic-framework MIL-53(Fe). J. Am. Chem. Soc. 2009, 131, 13002-13008.

(12) Bourrelly, S.; Llewellyn, P. L.; Serre, C.; Millange, F.; Loiseau, T.; Férey, G. Different adsorption behaviors of methane and carbon dioxide in the isotypic nanoporous metal terephthalates MIL-53 and MIL-47. J. Am. Chem. Soc. 2005, 127, 13519-13521.

(13) Serre, C.; Mellot-Draznieks, C.; Surblé, S.; Audebrand, N.; Férey, G. Role of solvent-host interactions that lead to very large swelling of hybrid frameworks. Science 2007, 30, 18281831.

(14) Bourrelly, S.; Moulin, B.; Rivera, A.; Maurin, G.; Devautour-Vinot, S.; Serre, C.; Devic, T.; Horcajada, P.; Vimont, A.; Clet, G.; Daturi, M.; Lavalley, J.-C.; Loera-Serna, S.; Denoyel, R.; Llewellyn, P. L.; Férey, G. Explanation of the adsorption of polar vapors in the highly flexible metal organic framework MIL-53(Cr). J. Am. Chem. Soc. 2010, 132, 9488-9498.

(15) Yang, X.; Camblor, M. A.; Lee, Y.; Liu, H.; Olson, D. H. Synthesis and crystal structure of as-synthesized and calcined pure silica zeolite ITQ-12. J. Am. Chem. Soc. 2004, 126, 10403-10409.

(16) Li, J.-R.; Kuppler, R. J.; Zhou, H.-C. Selective gas adsorption and separation in metalorganic frameworks. Chem. Soc. Rev. 2009, 38, 1477-1504. 
(17) Olson, D. H.; Yang, X.; Camblor, M. A. ITQ-12: a zeolite having temperature dependent adsorption selectivity and potential for propene separation. J. Phys. Chem. B 2004, 108, 11044-11048.

(18) Dubbeldam, D.; Torres-Knoop, A.; Walton, K. S. On the inner workings of monte carlo codes. Mol. Simul. 2013, 39, 1253-1292.

(19) Dubbeldam, D.; Calero, S.; Ellis, D. E.; Snurr, R. Q. RASPA: molecular simulation software for adsorption and diffusion in flexible nanoporous materials. Mol. Simul. 2016, $42,81-101$.

(20) Sanders, M. J.; Leslie, M.; Catlow, C. R. A. Interatomic potentials for $\mathrm{SiO}_{2}$. J. Chem. Soc., Chem. Commun. 1984, 1271-1273.

(21) Dubbeldam, D.; Torres-Knoop, A.; Walton, K. S. Force field parametrization through fitting on inflection points in isotherms. Phys. Rev. Lett. 2004, 93, 88302.

(22) Dubbeldam, D.; Calero, S.; Ellis, D. E.; Snurr, R. Q. United atom force field for alkanes in nanoporous materials. J. Phys. Chem. B 2004, 108, 12301-12313.

(23) Liu, B.; Smit, B.; Rey, F.; Valencia, S.; Calero, S. A new united atom force field for adsorption of alkenes in zeolites. J. Phys. Chem. C 2008, 112, 2492-2498.

(24) Peng, D.-Y.; Robinson, D. B. A new two-constant equation of state. Ind. Eng. Chem. Fundam. 1976, 15, 59-64.

(25) Nose, S. A molecular dynamics method for simulations in the canonical ensemble. Mol. Phys. 2002, 100, 191-198.

(26) Hoover, W. G. Canonical dynamics: equilibrium phase-space distributions. Phys. Rev. A 1985, 31, 1695-1697.

(27) Willems, T. F.; Rycroft, C. H.; Kazi, M.; Meza, J. C.; Haranczyk, M. Algorithms and tools 
for high-throughput geometry-based analysis of crystalline porous materials. Microporous Mesoporous Mater. 2012, 149, 134-141.

(28) Baerlocher, Ch.; McCusker, L. B. Database of zeolite structures: http://www.izastructure.org/databases/ (accessed Dec 2017).

(29) Rojas, A.; Martínez-Morales, E.; Zicovich-Wilson, C. M.; Camblor, M. A. Zeolite synthesis in fluoride media: structure direction toward ITW by small methylimidazolium cations. J. Am. Chem. Soc. 2012, 134, 2255-2263.

(30) Gutiérrez-Sevillano, J. J.; Dubbeldam, D.; Rey, F.; Valencia, S.; Palomino, M.; MartínCalvo, A.; Calero, S. Analysis of the ITQ-12 zeolite performance in propane-propylene separations using a combination of experiments and molecular simulations. J. Phys. Chem. C 2010, 114, 14907-14914.

(31) Metropolis, N.; Ulam, S. The monte carlo method. J. Am. Stat. Assoc. 1949, 44, 335-341. 


\section{TOC GRAPHICS}

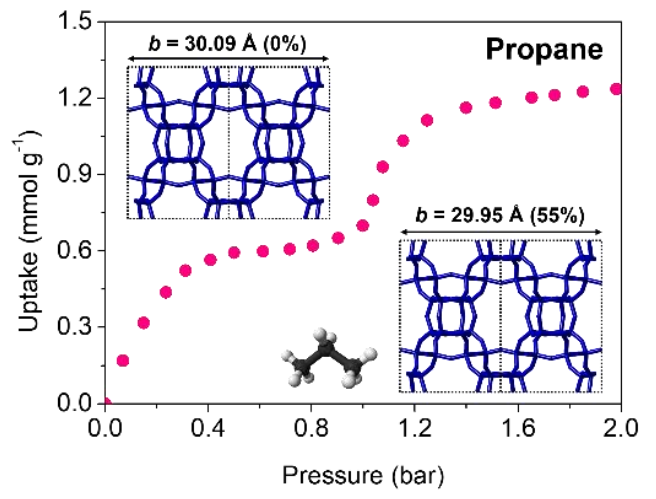

